\title{
Quantization and quantum-like phenomena: a number amplitude approach
}

\author{
T. R. Robinson \\ Department of Physics and Astronomy \\ University of Leicester, UK \\ e-mail: txr@le.ac.uk; tel: 0044 1162523562; fax: 00441162523555 \\ E. Haven \\ School of Management and IQSCS \\ University of Leicester, UK \\ e-mail: eh76@le.ac.uk; tel: 00441162523955
}

May 27, 2015

\begin{abstract}
Historically, quantization has meant turning the dynamical variables of classical mechanics that are represented by numbers into their corresponding operators. Thus the relationships between classical variables determine the relationships between the corresponding quantum mechanical operators. Here, we take a radically different approach to this conventional quantization procedure. Our approach does not rely on any relations based on classical Hamiltonian or Lagrangian mechanics nor on any canonical quantization relations, nor even on any preconceptions of particle trajectories in space and time. Instead we examine the symmetry properties of certain Hermitian operators with respect to phase changes. This introduces harmonic operators that can be identified with a variety of cyclic systems, from clocks to quantum fields. These operators are shown to have the characteristics of creation and annihilation operators that constitute the primitive fields of quantum field theory. Such an approach not only allows us to recover the Hamiltonian equations of classical mechanics and the Schrödinger wave equation from the fundamental quantization relations, but also, by freeing the quantum formalism from any physical connotation, makes it more directly applicable to non-physical, so-called quantum-like systems. Over the past decade or so, there has
\end{abstract}


been a rapid growth of interest in such applications. These include, the use of the Schrödinger equation in finance, second quantization and the number operator in social interactions, population dynamics and financial trading, and quantum probability models in cognitive processes and decision-making. In this paper we try to look beyond physical analogies to provide a foundational underpinning of such applications.

Keywords: quantization; quantum-like systems; number operator; quantum counting

\section{Introduction}

The importance of the theoretical framework of classical mechanics in early attempts to develop quantum theory in the mid to late 1920 s is well documented [1], [2]. Even in later stages of the search for a systematic quantum formalism, classical mechanics, particularly the elements involving Hamilton's equations and canonical variables, continued to influence the often intuitive developments. Heisenberg, in his famous paper of 1925 [3] had recognized the importance of replacing abelian variables of classical mechanics by non-abelian ones in quantum mechanics. Dirac cemented this view further with his idea of an analogy between the Poisson brackets of classical mechanics and the canonical commutation relation between non-commuting canonical variables in quantum theory [4], [5]. Canonical quantization became the focus of intense research by Jordan, Born, Dirac and others in the years 1926 and 1927 [6], which laid the essential theoretical foundations to much of the quantum mechanics and, what is considered to be, the even more fundamental quantum field theory that we know today.

Despite rapid progress made during that first intense burst of activity many unresolved questions remained, and the following decades saw a period of consolidation and reflection. Feynman, for example, while still recognizing the importance of classical mechanical relationships to quantum theory, was reportedly unhappy with Dirac's idea of an analogy only and sought a more direct connection [7]. Despite these concerns, Feynman was content to include classical concepts in quantum methods, as for example, when he directly incorporated the classical Lagrangian function into his revolutionary phase integral approach to quantum mechanics [8]. Wigner [9], from a different point of view from Feynman's, asked to what extent canonical quantization was essential, while retaining the Hamiltonian function of canonical variables from the classical theory. Wigner actually answered his own question, to some extent, by showing that there were alternatives to the usual 
canonical commutation relations, that were still consistent with Hamilton's equations. Wigner's question spawned new research into the significance of these alternative relations. Subsequent studies by Green [10], Greenberg and Messiah [11], and Kamefuchi and Takahashi [12] showed that these alternative relations were generalizations of the canonical forms that gave rise to particle descriptions that were neither purely fermionic nor purely bosonic and eventually lead to the notions of para-quantization and para-statistics [13]. One drawback of this theory is that such particle properties are not seen in nature. Moreover, Bialynicki-Birula [14] showed that these para-statistical fields could, in any case, be constructed from more fundamental ones. A number of other generalization procedures to the usual form of canonical commutation rules have also been considered. These include so-called qcalculus versions of the canonical quantization rule [15] that are consistent with q-derivatives and the pseudo-boson formalism [16] in which operators for different fields do not commute.

Despite many new developments in the formalism and understanding, there has still been a tendency to stick to a largely historical perspective in developing new quantum theory. This essentially consists of following the Dirac prescription of starting with a classical theory and then quantizing it. However, it has been pointed out that there is something intellectually unsatisfactory about this approach [13], [17] and that we should be starting with the more fundamental quantum field theory and recovering the classical. In this paper, we attempt to explore such a route. Here, as in the case of paraquantization, mentioned above, we do not presume canonical quantization, but unlike para-quantization, we do not presume classical mechanics either. That is to say we do not rely on Lagrangian or Hamiltonian formalism as a starting point. There are a number of motivations for this. One is to find a more intellectually satisfactory starting point for physical theory. Another is to see just how little one needs to assume, a priori, and still wind up with a recognizable physical theory. In particular we want to avoid the presumption of physical entities like particles and their space-time trajectories that are the foundation of classically based concepts. This then leaves us free to interpret our results in a way less constrained by our foundational assumptions. This is important since considerable interest has arisen, over the past decade or so, in the application of quantum formalism to non-physical systems, ranging, for example, from finance [18], [19] and population dynamics [20] to social science [21], psychology [22], cognition [23] and neuroscience [24]. Khrennikov [25] has coined the phrase quantum-like to describe such processes. However, there are legitimate questions to be posed about how applicable quantum methods are to non-physical systems that are not subject to the constraints placed on the physical world by say, the relationship between en- 
ergy and momentum and their corresponding conservation rules. Khrennikov [25] and Haven and Khrennikov [21] have argued that quantum mechanics is not essentially a physical theory. This paper goes some way to supporting such a view. We should like to stress that this work is not intended as a detailed investigation into the mathematical structure of quantum theory, but is, rather, a fresh look at some of its conceptual foundations.

We begin this exploration, in section 2, by introducing the mathematical preliminaries that involve a Hilbert space of vectors and operators on the vectors. In section 3 we outline the properties of vectors and operators as functions of a continuous variable (not necessarily of time) and the differentials of the vectors and operators. In section 4 we investigate the symmetry properties of operators that can be written as the product of an operator and its adjoint. Self-adjoint fields are introduced in section 5, from which the basic Hamiltonian equations of classical and quantum physics are recovered. The Schrödinger formalism is then recovered in section 6 . The overall results are summarized in section 7 , where we also briefly touch on some issues regarding applications to non-physical quantum-like systems.

\section{Mathematical preliminaries}

In this section we briefly outline the basic mathematical framework needed to represent the state of a system. By system, we do not necessarily mean anything physical and it certainly does not have to be thought of as a particle located in space and time. Rather it is simply something that can be characterized in a mathematical way. The basic mathematical elements that we need require no more than is to be found in almost any modern text book on quantum mechanics (see in particular the recent authoritative volume by Steven Weinberg [26] in which the development of the basic equations is based on symmetry arguments rather than on any analogies with classical mechanics).

Then, the state of a system is specified by a vector, $\Psi$, in a complex, Hilbert space, $H$. In briefly reviewing the essential mathematical developments we adopt Weinberg's mathematically more transparent notation than the Dirac notation that is traditionally used by physicists [27]. Then, if $\Psi, \Psi^{\prime}$ and $\Psi^{\prime \prime}$ are three vectors in the same space and $\alpha$ is a complex scalar, we can define an inner (scalar) product [28] of the form $\left(\Psi, \Psi^{\prime}\right)$, that is distributive over addition, $\left(\Psi, \Psi^{\prime}+\Psi^{\prime \prime}\right)=\left(\Psi, \Psi^{\prime}\right)+\left(\Psi, \Psi^{\prime \prime}\right)$, commutative with respect to scalar multiplication, $\left(\Psi, \alpha \Psi^{\prime}\right)=\alpha\left(\Psi, \Psi^{\prime}\right)$ and also has a symmetry property involving the complex conjugate, $\left(\Psi, \Psi^{\prime}\right)^{*}=\left(\Psi^{\prime}, \Psi\right)$. In addition, we can then identify $\|\Psi\|$ as the norm or modulus of $\Psi$ by $\|\Psi\|^{2}=(\Psi, \Psi)$, where 
$(\Psi, \Psi)>0$ as long as $\Psi$ is not a null vector.

This situation can be made more explicit by utilizing a complete set of orthogonal unit basis vectors, $\Psi_{i}$, to specify the space, such that any vector in the Hilbert space may be represented as

$$
\Psi=\sum_{i} \lambda_{i} \Psi_{i}
$$

where $\left(\Psi_{j}, \Psi_{i}\right)=\delta_{i j}$ and the $\lambda_{i}$ are complex numbers that are relate to $\Psi$ as follows: $\lambda_{i}=\left(\Psi_{i}, \Psi\right)$. Then

$$
\|\Psi\|^{2}=(\Psi, \Psi)=\sum_{i} \lambda_{i}^{*} \lambda_{i} .
$$

This shows that $(\Psi, \Psi)>0$, as long as $\Psi$ is not a null vector. In fact, in this case not all of the $\lambda_{i}$ are zero. The number of unit basis vectors in the Hilbert space is a measure of its dimensionality, which, in principle could be infinite.

Now consider a linear operator, $A$, operating on $\Psi$ that maps $\Psi$ to another vector, $\Phi$, in the same space, i.e.,

$$
A \Psi=\Phi .
$$

It is well known that if $A$ is defined on all of the vectors of $H$, i.e., if the domain of $A, D(A)$, coincides with $H$, then $A$ is bounded. However, in many interesting physical situations, $A$ turns out not to be everywhere defined, but still can be defined on some proper dense subset of $H$. This is the case when $A$ is unbounded. For instance the derivative operator is not defined for all square integrable functions, $L^{2}(\mathbb{R})$, but it is surely defined on a set $C^{\infty}$ of compactly supported functions that are dense in $L^{2}(\mathbb{R})$.

Following Weinberg's prescription, we can define the adjoint (if such exists), $A^{+}$of $A$ as that operator for which

$$
\left(\Phi, A^{+} \Psi\right)=(\Psi, A \Phi)^{*}=(A \Phi, \Psi) .
$$

Of course, Eq. (4) only makes sense for all $\Phi$ and $\Psi$ in $H$, if $A$ is bounded. In this case, also $A^{+}$is bounded, so is everywhere defined. On the other hand, if $A$ is unbounded, Eq. (4) only makes sense on suitable subsets of $H$, which are always assumed to be dense in $H$. In particular $\Phi$, must belong to $D(A)$ and to $\Psi$ to $D\left(A^{+}\right)$and both $D(A)$ and $D\left(A^{+}\right)$must be dense in $H$.

If $A$ is closed [28], it is a simple matter to show that $\left(A^{+}\right)^{+}=A$, and, $\left(\alpha A^{+}\right)=\alpha^{*} A^{+}$. Also, for a pair of operators, $A$ and $B$, operating sequentially, then, $(A B)^{+}=\left(B^{+} A^{+}\right)$, at least when these two sides are well defined. 
Again, while this is obvious whenever $A$ and $B$ are bounded, it is not necessarily true if $A$ and $B$ are unbounded. For instance, it may happen that given $\Psi$ is in $D(B)$, but $B \Psi$ is not in $D(A)$. When this happens, $B A \Psi$ is not well defined.

Before proceeding further, however, it is worth stressing that, even if we are encountering several complications whenever unbounded operators are considered, for many concrete applications these complications really disappear. This has been discussed, for instance, by Bagarello [20], who showed that, because of the existence of some integrals of motion attached to the system in question, the relevant unbounded operators are inevitably restricted to act in an effective, finite dimensional, Hilbert space whose explicit dimensionality depends on initial conditions. Hence, from now on, we will assume that all of the relevant operators are indeed bounded. However, we would like to add also that, even in different models where this is not possible, for instance in the absence of integrals of motion, the relevant unbounded operators involved are always at least densely defined, since they are combinations of lowering and raising operators that map the vectors of an orthonormal basis into other vectors of the same basis.

For a given operator $A$, it might happen that some special vectors exist that are mapped by $A$ into themselves. If $\Psi_{\alpha}$ is such a non-zero vector, then

$$
A \Psi_{\alpha}=\alpha \Psi_{\alpha},
$$

where $\alpha$ is, in general, a complex number. In this case, $\Psi_{\alpha}$ is called the eigenvector of $A$ and $\alpha$ is its eigenvalue. We also note that, in this case, if $\Psi_{\alpha}$ is a unit vector, such that $\left(\Psi_{\alpha}, \Psi_{\alpha}\right)=1$, then

$$
\left(\Psi_{\alpha}, A \Psi_{\alpha}\right)=\left(\Psi_{\alpha}, \alpha \Psi_{\alpha}\right)=\alpha\left(\Psi_{\alpha}, \Psi_{\alpha}\right)=\alpha .
$$

There are also special operators that are self-adjoint or Hermitian, such that $(\Phi, A \Psi)=(A \Phi, \Psi)$, for all $\Psi$ and $\Phi$ in $H$ (remember we are assuming $A$ is bounded). Then, if Eq. (5) applies, it follows that

$$
\left(\Psi_{\alpha}, A \Psi_{\alpha}\right)^{*}=\left(\Psi_{\alpha}, A \Psi_{\alpha}\right) .
$$

Hence $\alpha^{*}=\alpha$. Thus, as is well known, Hermitian operators have real eigenvalues. It is also well known that the converse is not true. There exist examples of non-Hermitian operators having real eigenvalues both for bounded and unbounded situations [29], [30].

A particularly relevant situation, interesting for what follows, arises when we consider a generic, possibly bounded, closed operator $Z$, which is not Hermitian. This can be used to construct a second operator, $Z^{+} Z$, which is 
in fact Hermitian and positive. Therefore, the eigenvalues of $Z^{+} Z$, if they exist, are unambiguously non-negative, and they are actually strictly positive if $Z$ is invertible. This non-negative property will prove important in what follows later. We next consider the differentiation of Hilbert space vectors that are functions of continuous variables.

\section{Functions of real, continuous variables and quantum differentiation}

We wish to explore some symmetry properties of the vectors and operators in the Hilbert space framework defined above. To do this we first assume that the vector $\Psi$ is a function, $\Psi(\xi)$, of a continuous real variable $\xi$. Then, assuming that the function $\Psi(\xi)$ is differentiable, we assume that the differential of $\Psi(\xi)$, with respect to $\xi$, is equal to a second vector, $\Phi(\xi)$, in the same Hilbert space, that is also a function of $\xi$, i.e. $\frac{d \Psi(\xi)}{d \xi}=\Phi(\xi)$. We also assume that these vectors are dense in the Hilbert space. We then seek an operator, $D_{\xi}$, on the Hilbert space, that maps from $\Psi(\xi)$ to $\Phi(\xi)$. Thus,

$$
\frac{d \Psi(\xi)}{d \xi}=D_{\xi} \Psi(\xi)
$$

Notice that, here, we are treating the mathematical expression $\frac{d \Psi(\xi)}{d \xi}$ as a single compound entity, a hieroglyph in some sense representing the function that is the derivative of $\Psi(\xi)$, and not the product of $\frac{d}{d \xi}$ with with $\Psi(\xi)$. Thus, in no sense is the symbol $\frac{d}{d \xi}$ equivalent to $D_{\xi}$. Also we can assume that $D_{\xi}$ is not itself a function of $\xi$.

Now we wish to explore a symmetry property of the norm of $\Psi(\xi)$ that leaves it unchanged when $\xi$ varies. This may seem a somewhat arbitrary and restrictive definition of $\xi$. However, the significance of this constraint will become clearer shortly. As we shall see, this property also leaves unchanged the general inner product of two vectors that are functions of $\xi$. The invariance of the norm with respect to changes in $\xi$ implies

$$
\left(\Psi(\xi), D_{\xi} \Psi(\xi)\right)+\left(D_{\xi} \Psi(\xi), \Psi(\xi)\right)=0 .
$$

Using the definition of adjoint and the distributive properties this leads to

$$
\begin{aligned}
\left(\Psi(\xi), D_{\xi} \Psi(\xi)\right)+\left(\Psi(\xi), D_{\xi}^{+} \Psi(\xi)\right) & = \\
\left(\Psi(\xi),\left(D_{\xi}^{+}+D_{\xi}\right) \Psi(\xi)\right) & =0,
\end{aligned}
$$


from which we can infer $D_{\xi}+D_{\xi}^{+}=0$, as long as $\Psi(\xi)$ is not null. Thus $D_{\xi}$ must be anti-Hermitian. It is convenient therefore to write $D_{\xi}=-i K_{\xi}$, where $K_{\xi}$ is a self-adjoint (Hermitian) operator. Thus,

$$
i \frac{d \Psi(\xi)}{d \xi}=K_{\xi} \Psi(\xi) .
$$

We can formally integrate Eq. (11) to yield $\Psi(\xi)=\exp \left(-i \xi K_{\xi}\right) \Psi(0)$, from which it is straightforward to show that

$$
(\Psi(\xi), \Psi(\xi))=(\Psi(0), \Psi(0)),
$$

as required. It is also clear why this form of dependence on $\xi$ does not alter the length of the Hilbert space vectors. It is because the functional dependence formally takes the form of a phase shift, albeit in operator form. In establishing Eq. (12) we need to use $\left(\exp \left(-i \xi K_{\xi}\right)\right)^{+}=\exp \left(i \xi K_{\xi}\right)$, which follows from expanding both sides in a power series in $\xi$ and equating term by term. It is also clear that Eq. (11) is quite generally applicable to any vector in the same Hilbert space that is a differentiable function of $\xi$. So, $\Phi(\xi)=$ $\exp \left(-i \xi K_{\xi}\right) \Phi(0)$, from which we can infer the invariance of $(\Psi(\xi), \Phi(\xi))$.

Now, suppose an operator $F$, which is itself not a function of $\xi$, maps $\Psi(\xi)$ to $\Phi(\xi)$, such that $F(0) \Psi(\xi)=\Phi(\xi)$, where the argument, 0 , emphasizes the fact that $F$ is not a function of $\xi$, then it follows that

$$
\left.(\Psi(\xi), F(0) \Psi(\xi))=\left(\Psi(0), \exp \left(i \xi K_{\xi}\right)\right) F(0) \exp \left(-i \xi K_{\xi}\right) \Psi(0)\right) .
$$

We can thus define a new operator $F(\xi)$ which is now an explicit function of $\xi$, such that

$$
F(\xi)=\exp \left(i \xi K_{\xi}\right) F(0) \exp \left(-i \xi K_{\xi}\right)
$$

Note that $F(\xi)$ only reduces to $F(0)$ if $F(0)$ commutes with $K_{\xi}$, which it generally is not assumed to do. Formal differentiation of $F(\xi)$ then yields

$$
i \frac{d F(\xi)}{d \xi}=F(\xi) K_{\xi}-K_{\xi} F(\xi)=\left[F(\xi), K_{\xi}\right] .
$$

The rule, Eq. (15) has a number of interesting properties that are worth noting. First, from the definition of adjoint, $F^{+}(\xi)=\exp \left(i \xi K_{\xi}\right) F^{+}(0) \exp \left(-i \xi K_{\xi}\right)$, which has exactly the same form as Eq. (14) and so the differential of the adjoint obeys Eq. (15) too. 
Second, the form of Eq. (15) is interesting from an algebraic point of view. The final (commutation) bracket in Eq. (15) is a Lie product. Using the antiHermitian $D_{\xi}$ instead of the Hermitian $K_{\xi}$, we can define a Lie product using a product symbol, $\wedge$, as

$$
D_{\xi} \wedge F(\xi)=D_{\xi} F(\xi)-F(\xi) D_{\xi}=\left[D_{\xi}, F(\xi)\right] .
$$

The product, $\wedge$, is not associative, i.e., if $G(\xi)$ is a second operator function of the same form as $F(\xi)$ in Eq. (14), then

$$
D_{\xi} \wedge(F(\xi) G(\xi))=\left(D_{\xi} \wedge F(\xi)\right) G(\xi)+F(\xi)\left(D_{\xi} \wedge G(\xi)\right),
$$

which defines an algebraic derivation and so automatically obeys Leibniz rule for the differentiation of a product. This works precisely because of the expansion rule of the Lie product, i.e.,

$$
\left[D_{\xi},(F(\xi) G(\xi))\right]=\left[\left(D_{\xi}, F(\xi)\right] G(\xi)-F(\xi)\left[\left(D_{\xi}, G(\xi)\right] .\right.\right.
$$

Note that this is true even when the associative rule applies to the product $D_{\xi} F(\xi) G(\xi)$, i.e., $D_{\xi}(F(\xi) G(\xi))=\left(D_{\xi}(F(\xi)) G(\xi)\right.$. Then, clearly, this product with $D_{\xi}$ is not a derivation, whereas $D_{\xi} \wedge$ is. Thus, we cannot have $\frac{d F}{d \xi} \neq D_{\xi} F$, but by contrast, $\frac{d F}{d \xi} \equiv D_{\xi} \wedge F$ is correct. This feature has important implications for the representation of operators by matrices and group algebra, whose products do obey an associative rule. Historically, Dirac [4] used an argument based on the relation between Lie products and Leibniz rule to deduce Eq. (15) directly for the differentiation of matrices.

We should emphasise that the operator, $K_{\xi}$, that appears in Eqs. (11) and (15), is not yet defined. When the formalism above is applied to idealized physical systems such as single particle dynamics, the continuous variable, $\xi$, is chosen to be time $t$. The corresponding operator in Eq. (11), $K_{t}$, is then conventionally identified with the particle energy operator. Eq. (11) is then of course the Schrödinger equation. Historically, this energy operator has been set equal to the Hamiltonian written as a function of the canonical variables, namely position coordinate and momentum, but with the classical scalar quantities replaced by a pair of non-commuting operators. This procedure is referred to as first quantization. The corresponding version of Eq. (15) is then referred to as Heisenberg's equation of motion. Here we do not follow this procedure and make no assumptions about $K_{\xi}$ based on canonical quantization, Hamiltonian mechanics nor on considerations of particle trajectories in space and time. Rather we apply these results to a general harmonic system, by which we mean some cyclic system with a sinusoidal variation, not necessarily in time. We introduce such a system as a symmetry property of certain Hermitian operators in the next section. 


\section{Symmetry, non-negativity and harmonic am- plitudes}

Consider an operator $J$ on vectors in a complex Hilbert space with an inner product as defined above. Assume that $J$ itself is invariant i.e., not a function of a real continuous variable, and that it is equal to the product of another operator $A$ on the same space and its adjoint, such that $J=A^{+} A$. As pointed out in section 2, we know that such an operator is not only Hermitian, but also has non-negative eigenvalues. We could assume $A$ also to be invariant in this sense. However we note that $J$ is invariant to a phase shift in $A$, i.e., $A \rightarrow \exp (-i \theta) A$, where $\theta$ is a (real) phase. Incidentally, it is important to distinguish between phase, which is a continuous variable with an infinite domain, as here, and a phase angle that is a representation of phase by the direction of a vector on a 2-dimensional plane. Then, because the directions of a vector with phase angles that differ by $2 \pi$ are indistinguishable, the values of the phase angle are conventionally restricted to an arbitrary domain of size $2 \pi$. What is really restricted of course is the amplitude of each of the components of the vector. Similarly, we do not regard time as restricted to a 12 hour domain simply because the position of the hands of a clock repeat every 12 hrs.

In order to explore the properties of this phase symmetry, we define a new operator $A(\theta)=\exp (-i \theta) A(0)$ that is a function of $\theta$. Of course we also have $A^{+}(\theta)=\exp (i \theta) A^{+}(0)$ and so $J=A^{+}(0) A(0)=A^{+}(\theta) A(\theta)$, as expected. Direct differentiation of $A(\theta)$ and $A^{+}(\theta)$ yields

$$
\frac{d A(\theta)}{d \theta}=-i A(\theta)
$$

and

$$
\frac{d A^{+}(\theta)}{d \theta}=i A^{+}(\theta)
$$

Applying Eq. (15) with $\xi=\theta$, gives

$$
A(\theta)=\left[A(\theta), K_{\theta}\right]
$$

and

$$
-A^{+}(\theta)=\left[A^{+}(\theta), K_{\theta}\right] .
$$

From Eqs. (21) and (22), it is a trivial matter to show that

$$
\frac{d\left(A^{+}(\theta) A(\theta)\right)}{d \theta}=\left[A^{+}(\theta) A(\theta), K_{\theta}\right]=0,
$$


with a similar result for $\frac{d\left(A(\theta) A^{+}(\theta)\right)}{d \theta}$.

It is interesting to note that Eqs. (21) and (22) may be obtained without differentiation, directly by equating

$$
A(\theta)=\exp (-i \theta) A(0)=\exp \left(i \theta K_{\theta}\right) A(0) \exp \left(-i \theta K_{\theta}\right),
$$

and then expanding the exponential functions as power series. Since Eq. (24) must be true for all $\theta$, then the power series must balance term by term. The first few orders yield

$$
\begin{aligned}
\theta^{0} & \Rightarrow A(0)=A(0), \\
\theta^{1} \Rightarrow A(0)=\left[A(0), K_{\theta}\right] & \\
\theta^{2} \Rightarrow & A(0)=\left[\left[A(0), K_{\theta}\right], K_{\theta}\right] \\
& \text { etc }
\end{aligned}
$$

Reassuringly, when the first order result is substituted into all of the higher order results, then they all give Eq. (21). But notice, Eq. (21) is satisfied by either $A(0)$ or $A(\theta)$. This is because $\left[A^{+}(0) A(0), K_{\theta}\right]=$ $\left[A^{+}(\theta) A(\theta), K_{\theta}\right]=0$. This is significant, bearing in mind that the form of $K_{\theta}$ has yet to be determined.

Thus far, no assumptions have been made about the properties of $K_{\theta}$. However, it is possible to infer certain properties from Eqs. (21) and (22). Let us define $\Psi_{\lambda}$ as a unit vector in a complex Hilbert space that is an eigenvector of $K_{\theta}$, with an eigenvalue of $\lambda$, i.e. $K_{\theta} \Psi_{\lambda}=\lambda \Psi_{\lambda}$. Then, from Eq. (21) we get

$$
K_{\theta}\left(A \Psi_{\lambda}\right)=A K_{\theta} \Psi_{\lambda}-A \Psi_{\lambda}=(\lambda-1) A \Psi_{\lambda} .
$$

Thus $A \Psi_{\lambda}$ is also an eigenvector of $K_{\theta}$, with an eigenvalue of $(\lambda-1)$, and must thus be proportional to $\Psi_{\lambda-1}$. Similarly, one finds that $A^{+} \Psi_{\lambda}$ is also an eigenvector of $K_{\theta}$, but this time with an eigenvalue of $(\lambda+1)$. Furthermore, $A^{2} \Psi_{\lambda}$ is an eigenvector of $K_{\theta}$ with an eigenvalue of $(\lambda-2)$. Obviously, repeated operation with $A$ on $\Psi_{\lambda}$ will yield a spectrum of eigenvalues for $K_{\theta}$, that has the form $\left(\lambda_{0}+n\right)$, where $\lambda_{0}$ is an ordinary number that is independent of $\Psi_{\lambda}$, and $n$ is an integer whose values depend on the eigenvector. Let us call the operator on the same Hilbert space with these eigenvectors and with this eigenvalue spectrum, $L$, i.e. $K_{\theta}=L$, and relable the eigenvectors $\Psi_{\lambda} \rightarrow \Psi_{n}$. So $L \Psi_{n}=\left(\lambda_{0}+n\right) \Psi_{n}$.

At this point there is no constraint on what integer value $n$ can have, so its value can run through both positive and negative integers as well as zero, since $L$ is not yet fully defined. However, we can infer, from Eqs. 
(21) and (22) that both $A^{+} A$ and $A A^{+}$commute with $L$ and can thus each be some arbitrary function of $L$. To be specific, and noting that we are interested mainly in the integer eigenvalues, we can let $A^{+} A=f\left(L-\lambda_{0} I\right)$, where $I$ is the identity operator and $f\left(L-\lambda_{0} I\right)$ is some arbitrary function of $L-\lambda_{0} I$, defined in the usual way [26], i.e. since $\left(L-\lambda_{0} I\right) \Psi_{n}=n \Psi_{n}$, then $f\left(L-\lambda_{0} I\right) \Psi_{n}=f(n) \Psi_{n}$. To be clear the symbol, $f$, here, is neutral, in the sense that, if its argument is an operator, then the function is an operator also, while if the argument is a scalar, then the function is a scalar. Thus, $f\left(L-\lambda_{0} I\right)$ is an operator while $f(n)$ is a scalar. The precise form of $f$ remains undetermined in this case, but we can infer that $A A^{+}=f(L+$ $\left.I-\lambda_{0} I\right)$, as follows. If we let $A \Psi_{n}=g(n) \Psi_{n-1}$ and $A^{+} \Psi_{n}=h(n) \Psi_{n+1}$, where $g(n)$ and $h(n)$ can be assumed to be real without loss of generality, then $\left(\Psi_{n}, A^{+} A \Psi_{n}\right)=h(n-1) g(n)$. But $\left(\Psi_{n}, A^{+} A \Psi_{n}\right)=\left\|A \Psi_{n}\right\|^{2}=g^{2}(n)$, so we must have, $h(n-1)=g(n)$. Writing $g^{2}(n)=f(n)$ then yields the desired result [see ref. 13]. As we have seen, these relations also reflect the fact that, from Eqs. (21) and (22), both $A^{+} A$ and $A A^{+}$commute with $L$ and hence are functions of $L$. However, even though the relations $A^{+} A=f\left(L-\lambda_{0} I\right)$ and $A A^{+}=f\left(L+I-\lambda_{0} I\right)$ imply that $f\left(L-\lambda_{0} I\right)$ and $f\left(L+I-\lambda_{0} I\right)$ must have non-negative eigenvalues, this still does not force $L$ itself, necessarily, to have non-negative eigenvalues.

As already mentioned, if we were dealing with physical systems as functions of time, then, conventionally, we would associate $L$ with the energy operator. Here we make no such assumption, but simply postulate that the spectrum of eigenvalues of $L$ should be non-negative. This implies that $\left(\lambda_{0}+n\right) \geq 0$. If we take $\lambda_{0}$ to be a positive constant, then $n$ can run through all of the non-negative integer values from zero to infinity. We can then represent $L$ as $N+\lambda_{0}$, where the operator $N$ has an eigenvalue spectrum corresponding to the non-negative integers, i.e., $N \Psi_{n}=n \Psi_{n}$, where $n \geq 0$.

Because of this non-negative property identified in section 2 above, we can further infer that $N$ itself can be expressed in terms of a closed number amplitude operator $Z$ and its adjoint, $Z^{+}$, such that $N=Z Z^{+}$, since, $\left(\Psi_{n}, N \Psi_{n}\right)=\left\|Z \Psi_{n}\right\|^{2}=n$. It should be emphasized that here, $N=Z^{+} Z$, is regarded as essentially defining $Z$, to within the arbitrary factor $\exp (-i \theta)$. Then we can again use the symmetry, $Z(\theta)=\exp (-i \theta) Z(0)$ and hence, $N=Z^{+}(0) Z(0)=Z^{+}(\theta) Z(\theta)$. Then, applying Eqs. (21) and (22), to $Z$ and $Z^{+}$, with $K_{\theta}=L=N+\lambda_{0} I$, produces

$$
Z=[Z, N]=\left[Z, Z^{+} Z\right]
$$

and 


$$
-Z^{+}=\left[Z^{+}, N\right]=\left[Z^{+}, Z^{+} Z\right],
$$

where we have used the fact that $\left[Z, \lambda_{0}\right]=\left[Z^{+}, \lambda_{0}\right]=0$. In this case, from the arguments above we can deduce the form of $f(N)$, as follows. Since $Z^{+} Z=f(N)=N$, then $Z Z^{+}=f(N+I)=N+I$, and hence ${ }^{1}$

$$
\left[Z, Z^{+}\right]=I \text {. }
$$

The above results may be obtained in an even more telling way as follows. Since $Z$ and $Z^{+}$are clearly non-abelian we could have started with $N=Z Z^{+}$ instead of $N=Z^{+} Z$, since to begin with, $\left[Z, Z^{+}\right]$is not known. Indeed, in that case, the most general form for $N$ should contain a combination of $Z^{+} Z$ and $Z Z^{+}$. Defining $\lambda_{0}=\alpha_{0}+\beta_{0}$, where $\alpha_{0}$ and $\beta_{0}$ are two arbitrarily chosen non-negative numbers, apart from the fact that we will insist that $0 \leq \beta_{0} \leq 1$, we can write

$$
N+\beta_{0} I=S^{2} Z^{+} Z+C^{2} Z Z^{+}=S^{2} f(N)+C^{2} f(N+I),
$$

where $S$ and $C$ are real numbers. Now we can always scale the right hand side of Eq. (30) by dividing by $S^{2}+C^{2}$, so we can quite generally assume that $S=\sin \gamma$ and $C=\cos \gamma$ where $\gamma$ is some arbitrary angle. Operating with Eq. (30) on $\Psi_{n}$ and rearranging leads to the iterative relation

$$
f(n+1)=\left(\beta_{0}+n\right) \sec ^{2} \gamma-f(n) \tan ^{2} \gamma .
$$

Taking $f(0)=0$, then leads to

$$
f(n)=\sec ^{2} \gamma \sum_{m=1}^{n}\left(\left(\beta_{0}+n-m\right)\left(-\tan ^{2} \gamma\right)^{m-1}\right) .
$$

Thus, after a little manipulation

$$
f(n+1)-f(n)=1+\left(\beta_{0} \sec ^{2} \gamma-1\right)\left(-\tan ^{2} \gamma\right)^{n} .
$$

Eq. (33) implies that $\left[Z, Z^{+}\right]$appears to be generally dependent on $n$ and hence is not a fixed number times $I$. However, since both $\beta_{0}$ and $\gamma$ are quite arbitrary, we can choose, without loss of generality, to set $\beta_{0}=\cos ^{2} \gamma$. Then we get $f(n+1)-f(n)=1$, which recovers $\left[Z, Z^{+}\right]=I$ quite generally. We also then have

$$
N=\sin ^{2} \gamma Z^{+} Z+\cos ^{2} \gamma Z Z^{+}-\cos ^{2} \gamma I=Z^{+} Z .
$$

\footnotetext{
${ }^{1}$ Notice also, that since $N$ is an unbounded operator then so are $Z$ and $Z^{+}$. Thus, properly speaking, we should have $\left[Z, Z^{+}\right]^{++}=I$, where ${ }^{++}$denotes closure.
} 
So remarkably, $N=Z^{+} Z$ is quite general after all, and the self-adjoint version of the phase differential operator $L$ is just

$$
L=N+\lambda_{0} I=Z^{+} Z+\lambda_{0} I .
$$

The above result implies $f(n)=n$. Also, recalling that $f(n)=g^{2}(n)$, where $Z \Psi_{n}=g(n) \Psi_{n-1}$, leads directly to the number amplitude operator, $Z$ and its conjugate having the well-known properties of ladder operators, i.e.

$$
Z \Psi_{n}=\sqrt{n} \Psi_{n-1} \text { and } Z^{+} \Psi_{n}=\sqrt{n+1} \Psi_{n+1}
$$

and hence that $Z \Psi_{0}=0$, so that the eigenvectors of $N$ have the expected lower bound, with the eigenvector, $\Psi_{0}$. As is well-known, the ladder operators in Eq. (36) are interpreted as creation and annihilation operators in quantum field theory and constitute a primitive field. The excitation of this field is interpreted in terms of particles and $\Psi_{0}$ the vacuum state with no particles in the context of quantum field theory. The condition, $Z \Psi_{0}=0$, implied by Eq. (36), effectively shuts off access to the negative integers in the spectrum that would destroy the non-negative property of $N$ and $L$. The existence of this terminating vacuum state is thus essential.

Below we outline how the basic laws of classical mechanics and also of Schrödinger wave mechanics can be recovered from Eqs. (29) and (35). First we need to separate the creation and annihilation operators into their selfadjoint parts.

\section{Self-adjoint fields and Hamiltonian mechan- ics}

Here we examine the consequences of replacing the pair $Z^{+}$and $Z$ in Eq. (35) by a pair of Hermitian variables $X$ and $Y$, defined by

$$
X=\frac{\left(Z^{+}+Z\right)}{\sqrt{2}} \text { and } Y=\frac{i\left(Z^{+}-Z\right)}{\sqrt{2}} .
$$

The self-adjoint nature of variables $X$ and $Y$, can be seen by noting that $Z$ is closed and then that, from Eq. (37), and $X^{+}=X$ and $Y^{+}=Y$. L may now be written

$$
L=\left(X^{2}+Y^{2}+i(X Y-Y X) / 2\right)+\lambda_{0} I .
$$

Now Eq. (29) leads to 


$$
X Y-Y X=[X, Y]=i I
$$

and so

$$
L=\left(X^{2}+Y^{2}-I\right) / 2+\lambda_{0} I
$$

At this point, in order to establish a connection with Hamiltonian mechanics it is convenient to introduce time, $t$. This is simply done by replacing phase by $\theta=\omega t$, where $\omega$ is an arbitrary scaling factor with dimensions of angular frequency. Here the time merely serves to parameterize the phase. Then $D_{t}=\omega D_{\theta}$ and we can define a frequency operator $\Omega=\omega L$. Then

$$
\Omega=\omega\left(Z^{+} Z+\lambda_{0} I\right)=\omega\left(N+\lambda_{0} I\right)=\omega\left(X^{2}+Y^{2}+2 \lambda_{0} I-I\right) / 2 .
$$

Eq. (41) could be put into standard Hamiltonian form by multiplying it by $\hbar$ and choosing $\lambda_{0}=1 / 2$, but we can just as well take Eq. (41) as a scaled Hamiltonian operator in what follows. Then $X$ and $Y$ can be interpreted as a pair of real bosonic fields, such as those that comprise the electromagnetic field or a mechanical oscillator. In the Hamiltonian context they represent a pair of generalized co-ordinates.

Having obtained the basic equations of quantum field theory and the field relations without the aid of classical mechanics, we are now free to derive the equations of classical mechanics from the forgoing rules. First, the time derivatives of the self-adjoint, bosonic fields $X$ and $Y$ obey

$$
i \frac{d X}{d t}=[X, \Omega]
$$

and

$$
i \frac{d Y}{d t}=[Y, \Omega]
$$

We note from Eq. (41) that $\Omega$ separates into two functions, $\Omega(X, Y)=$ $T(Y)+V(X)$, plus some constant terms, where $T(Y)=\omega Y^{2} / 2$ and $V(X)=$ $\omega X^{2} / 2$. By iterative application of the expansion rule of commutation brackets, $[A, B C]=B[A, C]+[A, B] C$, one finds that Eq. (39) leads to $[X, f(Y)]=$ $i \frac{\partial f}{\partial Y}$ and $[Y, f(X)]=-i \frac{\partial f}{\partial X}$, assuming the functions are analytical and can be expressed as infinite Taylor series. Thus,

$$
\frac{d X}{d t}=\frac{\partial \Omega}{\partial Y}
$$

and 


$$
\frac{d Y}{d t}=-\frac{\partial \Omega}{\partial X}
$$

which are recognizable as the Hamilton equations of classical mechanics, with $X$ and $Y$ playing the roles of the canonical coordinate and the canonical momentum, respectively. Notice that, Eqs. (44) and (45), as well as the commutation relation Eq. (39), remain true even when $T(Y)$ and especially $V(X)$ are generalized to non-harmonic systems. Then Eq. (45) becomes

$$
\frac{d Y}{d t}=-\frac{\partial V(X)}{\partial X}
$$

which has the form of Newton's second law of motion. This generalization is the basis of classical Hamiltonian mechanics with an appropriate potential function, $V(X)$. $\Omega$ is then the classical Hamiltonian and the equations of motion are obtained from Eqs. (44) and (45). It is important to point out here that the derivation of equations of classical mechanics described here rests entirely on the result that $\left[Z, Z^{+}\right]=I$.

There is a clear distinction between the type of dynamics associated with a free field that is characterized by a Hamiltonian of the form Eq. (41) and the free particle of traditional classical (Newtonian or Lagrangian) mechanics where $V(X)=0$ and then the canonical momentum is constant in time. So, for physical systems at least, the free field is associated with cyclic motion and the free particle with uniform rectilinear motion.

Eqs. (44) and (45) apply equally well to classical or quantum mechanical systems. Of course, the fundamental difference between classical and quantum systems is that the former are represented by abelian (commuting) scalar variables and the latter by non-abelian (non-commuting) variables. This leads to a major difference in the way information about the system in question is obtained. We need to bear in mind that information is itself a scalar quantity. For classical systems the observables are scalars and can be measured directly to yield quantitative information. By contrast, to obtain information about the observables of a quantum system the non-abelian variables are treated as operators. Then, if the operator representing the observable is $Q$, the quantitative information about such a system is given by an expectation value that is equal to $\operatorname{Tr}(\rho Q)[21],[26]$, where $\rho$ is the density operator for the state of the system; $Q$ may or may not have eigenvalues, but is self-adjoint.

Formally, it is a straightforward matter to distinguish, in a quantitative way, between quantum and classical systems. In the former, the canonical variables do not commute, while in the latter they do. If we define a degree of non-commutivity, $\Delta$, for variables $X$ and $Y$ as the ratio of size of expectation 
value of their commutation bracket to the product of the root-mean-square values,

$$
\Delta=\frac{|<[X, Y]>|}{\sqrt{<X^{2}><Y^{2}>}}
$$

where $\langle X\rangle=\left(\Psi_{n}, X \Psi_{n}\right)$ etc., then we expect classical behaviour when $\Delta$ is small and so $X$ and $Y$ can be treated as ordinary commuting variables. $\Delta$ in Eq. (47) is easily evaluated to be $1 /(n+1 / 2)$. So, the system will behave classically when $n$ is very large. The meaning of this result depends of course on specific interpretations of the number operator. It is usually interpreted as representing the number of particles in a state. For example, if $X$ and $Y$ represent a monochromatic electromagnetic field, then $n$ is the number of photons of a particular frequency. This field may be treated classically when the number of photons is very large.

\section{The Schrödinger picture}

It is also a straightforward matter to recover the Schrödinger form of quantum theory from the foregoing results. From Eq. (11), we choose $\xi=t$, where $t$ is time. Then with the scaled Hamiltonian operator, $\Omega$, from the previous section, we obtain the well-known Schrödinger equation

$$
i \frac{\partial \Psi(t)}{\partial t}=\Omega \Psi(t)=(T(Y)+V(X)) \Psi(t) .
$$

To complete the Schrödinger formalism, $X$ can be retained from the previous section, but to obtain the momentum in Schrödinger formalism, we need to convert $Y$ to a differential form that still satisfies the commutation bracket, Eq. (39). We do this by noting that $Y$ in Eq. (39) is formally isomorphic with $\frac{-i \partial}{\partial X}$, so that $\Omega$ becomes

$$
\Omega=-\left(\frac{\omega}{2}\right)\left(\frac{\partial^{2}}{\partial X^{2}}\right)+V(X) .
$$

It is also worth commenting here that we have obtained the Schrödinger equation without any real concern for space and time. Time is only included at all as a parameterization of phase and only then to make a connection to the conventional form of the Hamilton equations of mechanics. There has certainly been no need for considerations of relativistic versus non-relativistic particle motion of any kind. This is quite different from conventional approaches in which the Schrödinger equation appears as the non-relativistic limit of the Klein-Gordon equation. 


\section{Conclusions}

In this paper, we have obtained the properties, Eqs. (29), (45) and (46), of the number amplitude operator, $Z$ and its adjoint $Z^{+}$, that are interpreted as creation and annihilation operators in the second quantization formalism, and that also serve as the primitive quantum fields of the theory. This was done on the basis of an invariant Hilbert space representation, without presuming any of the formalism of classical mechanics, particularly the Lagrangian and Hamiltonian formalisms that are the basis of most approaches to second quantization. Thus, any notion of particles and particle trajectories in space and time are absent from our starting assumptions. So, contrary to the conventional procedure, we obtained some key equations of quantum physics without quantizing an already known classical physics theory. Indeed, we would claim that the classical theory follows from our quantum one. Instead, the results have been obtained by restricting the eigenvalue spectrum of the differential operator with respect to phase, $K_{\theta}$, which, when applied to a harmonic field consists of integers, to non-negative values, i.e. to the counting numbers including zero. The non-negative assumption applies essentially to the eigenvalue spectrum of the number operator $N$ and this allows us to define it as $N=Z^{+} Z$. The operator $Z$ can be taken as a function of phase, $\theta$, such that $Z(\theta)=\exp (-i \theta) Z(0)$ and can represent any cyclic system, not necessarily a physical one. Furthermore, from the commutation relation that results, between $Z$ and $Z^{+}$, we obtained the commutation rule between their self-adjoint components, $X$ and $Y$, that represent a pair of canonical variables. This commutation rule then led directly to the Hamilton equations of classical and quantum mechanics. Thus we achieved a logical progression from the more fundamental quantum field theory to classical mechanics and the Schrödinger equation.

A further interesting result of our approach is that no distinction needs to be drawn between first and second quantization. Conventionally, second quantization involves promoting the complex wave functions that obey the Schrödinger equation, together with their complex conjugates, to non-abelian operators. According to Darrigol [31], this was first suggested by Jordan and Klein [32], using somewhat ad hoc arguments. In our approach, having established second quantization rules directly from operator algebra, we are free to develop the Schrödinger formalism by introducing the representation of the non-abelian canonical variables by non-commuting co-ordinate and conventional differential operator pairs. The wave functions remain simply as eigenfunctions in both forms of quantization. Thus, there is really only one type of quantization.

As stated above, our results have been obtained without quantizing any 
classical physical rules. If anything was quantized in our approach, then it was classical counting. By classical counting we mean that the counting numbers, $n=0,1,2,3, \ldots$, are simply scalars. The quantum equivalent involves representing counting numbers using the number amplitude operators, $Z$ and $Z^{+}$, and the number operator, $N=Z^{+} Z$, that has a spectrum of eigenvalues equal to the counting numbers, including zero. We could then describe this formulation as quantum counting. This connection between our quantum counting and classical counting is then analogous to the connection between quantum and classical representations of probabilities. Classical probability is regarded as a scalar, $P$, that obeys the classical Kolmogorov rules, whereas quantum probability is formulated in terms of a probability density amplitude that is represented by a quantum wavefunction, $\phi$, which is subject to the rules of quantum mechanics, such that the probability density equals $\phi^{*} \phi$.

We believe our results have also gone some way to support the contention of Khrennikov [25] and Haven and Khrennikov [21], that quantum theory is not essentially a physical theory, although of course it does necessarily reproduce the equations that are applicable to physical systems too. Khrennikov has coined the term quantum-like, for phenomena describable in terms of quantum probability density amplitudes (wave functions). We have shown above that the counting numbers, including zero, appear to play a special role in the emergence of second quantization methods. Counting numbers imply the notion of objects to be counted in the broadest sense. Phenomena that can be represented by this quantum counting formalism involving $Z$ and $Z^{+}$, could, by analogy with Khrennikov, be termed quantum-field-like. Bagarello [20], [33] has recently treated population number dynamics and finance using second quantization techniques and the number operator. This work has recently been developed further to include information exchange [34].

A detailed discussion of the applications of the present formalism to quantum-like phenomena is beyond the scope of the present paper. A generalization to multi-mode harmonic systems and the interaction and coupling between these modes, among other things, is needed. This coupling is important in the interpretation of the potential function introduced in section 5. It turns out that this generalization of the formalism presented above is actually quite straightforward, as is its extension from, what are here, essentially bosonic systems, to fermionic ones. These issues will form the basis of a future publication. 


\section{Acknowledgements}

The authors are sincerely grateful to Fabio Bagarello for his help in clarifying a number of the mathematical technicalities that arise in the paper. Thanks are also due to an unknown referee for his valuable comments on the structure of the paper.

\section{References}

[1] Lacki, J.: The puzzle of canonical transformations in early quantum mechanics. Stud. Hist. Philos. M. P. 35, 317-344 (2004)

[2] Duncan, A., Janssen, M.: From canonical transformations to transformation theory, 1926-1927: The road to Jordan's Neue Begründung. Stud. Hist. Philos. M. P. 40, 352-362 (2009)

[3] Heisenberg, W.: Über die quantentheoretische Umdeutung kinematischer und mechanischer Beziehungen. Z. Phys. 33, 879-893 (1925)

[4] Dirac, P.A.M.: The fundamental equations of quantum mechanics. Proc. Roy. Soc. Lond. A 109, 642-653 (1925)

[5] Dirac, P.A.M.: On the theory of quantum mechanics. Proc. Roy. Soc. Lond. A 112, 661-677 (1926)

[6] van der Waerden, B. L.: Sources of quantum mechanics. Dover, New York (2007)

[7] Derbes, D.: Feynman's derivation of Schrödinger's equation. Am. J. Phys. 64, 881-884 (1995)

[8] Feynman, R. P.: Space-time approach to non-relativistic quantum mechanics. Rev. Mod. Phys. 20, 367-387 (1948)

[9] Wigner, E. P.: Do the equations of motion determine the quantum mechanical commutation relations? Phys. Rev. 77, 711-712 (1950)

[10] Green, H. S.: A generalized method of field quantization. Phys. Rev. 90, 270-273 (1953)

[11] Greenberg, O. W., Messiah, A.M.L.: Selection rules for parafields and the absence of parafields in nature. Phys. Rev. 138, B1155-B1167 (1965) 
[12] Kamefuchi, S., Takahashi, Y.: A generalization of field quantization and statistics. Nucl. Phys. 36, 177-206 (1962)

[13] Ohnuki, Y., Kamefuchi, S.: Quantum field theory and parastatistics. University of Tokyo Press, Tokyo (1982)

[14] Bialynicki-Birula, I.: Elementary particles and generalized statistics. Nucl. Phys. 49, 605-608 (1963)

[15] Swamy, P. N.: Deformed Heisenberg algebra: origin of q-calculus. Physica A 328, 145-153 (2003)

[16] Bagarello, F.: Pseudobosons, Riesz bases, and coherent states. J. Math. Phys. 51, 023531 (2010)

[17] Wallace, D.: In defence of naiveté: The conceptual status of Lagrangian quantum field theory. Synthese 151, 33-80 (2006)

[18] Baaquie, B. E.: Quantum Finance, Cambridge University Press, Cambridge, (2004)

[19] Haven, E.: Pilot wave theory and financial option pricing, Int. J. Th. Phys. 44, 1957-1962 (2005)

[20] Bagarello, F.: Quantum Dynamics for Classical Systems, Wiley, New York, (2013)

[21] Haven, E., Khrennikov, A.: Quantum Social Science, Cambridge University Press, Cambridge (2013)

[22] Aerts, D.: Quantum structure in cognition, J. Math. Psychol. 53, 314$348(2009)$

[23] Busemeyer, J. R., Bruza, P. D.: Quantum models of Cognition and Decision, Cambridge University Press, Cambridge (2012)

[24] Khrennikov, A. Yu.: The quantum-like brain on the cognitive and subcognitive time scales, J. Consciousness Stud. 15, 39-77 (2008)

[25] Khrennikov, A. Yu.: Ubiquitous Quantum Structures, Springer-Verlag, Berlin (2010)

[26] Weinberg, S.: Lectures in quantum mechanics, Cambridge University Press, Cambridge (2013) 
[27] Dirac, P.A.M.: The Principles of Quantum Mechanics (4e). Oxford University Press, Oxford (1958)

[28] Reed, M., Simon, B.: Methods in modern mathematical physics, vol. I, Functional analysis, Academic press, Boston (1980)

[29] Bagarello, F.: From self-adjoint to non self-adjoint harmonic oscillators: physical consequences and mathematical pitfalls. Phys. Rev. A 88, $032120(2013)$

[30] Bagarello, F.: Model pseudofermionic systems: connections with exceptional points. Phys. Rev. A 89, 032113 (2014)

[31] Darrigol, O.: The origin of quantized matter waves. Hist. Stud. Phys. Sci. 16, 197-253 (1986)

[32] Jordan, P., Klein, O.: Zum Mehrkörperproblem der Quantentheorie. Z. Phys. 45, 751-765 (1927)

[33] Bagarello, F.: Stock markets and quantum dynamics: A second quantized description, Physica A, 386, 283-302 (2007)

[34] Bagarello, F., Haven, E.: Towards a formalization of a two trader market with information exchange, Phys. Scripta 90, 015203 (2015) 